\title{
Recurrence Techniques for the Calculation of Bessel Functions
}

\author{
By M. Goldstein and R. M. Thaler
}

1. Introduction. The Bessel functions lend themselves most readily to calculation by recurrence techniques [1]. Let us consider the regular and irregular Bessel function of real order and argument $J_{\nu}(x)$ and $Y_{\nu}(x)$. These functions both obey the same recurrence relation, viz.

$$
F_{\nu-1}(x)+F_{\nu+1}(x)=\frac{2 \nu}{x} F_{\nu}(x)
$$

where $F_{\nu}(x)$ may be either $J_{\nu}(x)$ or $Y_{\nu}(x)$. If one is given $Y_{\nu}(x)$ and $Y_{\nu+1}(x)$ then Eq. (1) may be used to generate the functions $Y_{\nu+n}(x)$. For $\mu \gg(x / 2)$ the function $Y_{\mu}(x)$ increases extremely rapidly with increasing order, i.e., $Y_{\mu}(x) \sim(2 \mu / x)^{\mu}$ and the functions $Y_{\nu+n}(x)$ calculated from Eq. (1) yield good accuracy for large $n$.

However, if one is given $J_{\nu}(x)$ and $J_{\nu+1}(x)$, Eq. (1) gives poor accuracy for $J_{\nu+n}(x)$, since for $\mu \gg(x / 2), J_{\mu}(x) \sim(2 \mu / x)^{-\mu}$. On the other hand, if one is given $J_{\nu+n}(x)$ and $J_{\nu+n+1}(x)$, where $n \gg(x / 2)$, then one may again recur without loss of accuracy but this time in the direction of decreasing order. We shall first treat the problem of using the recurrence technique in the calculation of the regular Bessel function $J_{\nu+n}(x)$. Thus, let us find $J_{\nu+n}(x)$, for $0 \leqq \nu<1$ and $n \leqq N$.

2. The Regular Bessel Function. Consider a function $F_{\nu+n}(x)$, which obeys Eq. (1), and defined such that

$$
\begin{array}{r}
F_{\nu+M+1}(x)=0 \\
F_{\nu+M}(x)=a
\end{array}
$$

where $a$ may be chosen to be any constant, and $M \gg N$. By successive application of the recurrence relation, Eq. (1), we may now generate $F_{\nu+M-1}(x), \cdots, F_{\nu}(x)$.

Since $F_{\nu+M+1}(x)$ and $F_{\nu+M}(x)$ can be treated as the same linear combination of the regular and irregular Bessel functions, then

$$
\begin{aligned}
F_{\nu+M+1}(x) & =\alpha J_{\nu+M+1}(x)+\beta Y_{\nu+M+1}(x), \\
F_{\nu+M}(x) & =\alpha J_{\nu+M}(x)+\beta Y_{\nu+M}(x),
\end{aligned}
$$

and, in general,

$$
F_{\nu+n}(x)=\alpha J_{\nu+n}(x)+\beta Y_{\nu+n}(x)
$$

so that

$$
F_{\nu+M}(x)=\alpha J_{\nu+M}(x)\left[1+\frac{\beta}{\alpha} \frac{Y_{\nu+M}(x)}{J_{\nu+M}(x)}\right],
$$

Received Jan. 12, 1959. Work performed under the auspices of the U. S. Atomic Energy Commission. 
and

$$
F_{\nu+N}(x)=\alpha J_{\nu+N}(x)\left[1+\frac{\beta}{\alpha} \frac{Y_{\nu+N}(x)}{J_{\nu+N}(x)}\right] .
$$

Since $M$ is chosen such that $M \gg N$, then it is clear that

$$
\frac{\frac{\beta}{\alpha} \frac{Y_{\nu+N}(x)}{J_{\nu+N}(x)}}{\frac{\beta}{\alpha} \frac{Y_{\nu+M}(x)}{J_{\nu+M}(x)}}=\left(\frac{Y_{\nu+N}(x)}{Y_{\nu+M}(x)}\right)\left(\frac{J_{\nu+M}(x)}{J_{\nu+N}(x)}\right) \ll 1,
$$

so that for $n \leqq N$

$$
F_{\nu+n}(x) \cong \alpha J_{\nu+n}(x)
$$

Clearly $M$ may be chosen so large that $F_{v+n}(x)=\alpha J_{\nu+n}(x)$ to any desired numerical accuracy.

Determination of $\alpha$ will then yield the regular Bessel function $J_{\nu+n}(x)$ for $n \leqq N$. To do this one may make use of one of several addition theorems; for example, the addition theorem [2]:

$$
2^{\nu} \sum_{m=0}^{\infty}(\nu+2 m) J_{\nu+2 m}(x) x^{-\nu} \Gamma(\nu+m) / m !=1 .
$$

For $\nu=0$ Eq. (8) reduces to the familiar result

$$
J_{0}(x)+2 \sum_{m=1}^{\infty} J_{2 m}(x)=1 .
$$

To any given accuracy there exists an even integer $L$, such that

$$
2^{\nu} \sum_{m=0}^{L / 2}(\nu+2 m) J_{\nu+2 m}(x) x^{-\nu} \Gamma(\nu+m) / m ! \cong 1 .
$$

If $L \leqq N$, then to the desired accuracy one may write

$$
2^{\nu} \sum_{m=0}^{L / 2}(\nu+2 m) F_{\nu+2 m}(x) x^{-\nu} \Gamma(\nu+m) / m ! \cong \alpha .
$$

If $L>N$, the desired accuracy may nevertheless be obtained by increasing $M$. Eq. (11) may be rewritten in a form more suitable for numerical computation as:

$$
\sum_{m=0}^{L / 2} \phi_{m} F_{v+2 m}(x)=\alpha,
$$

where

$$
\begin{aligned}
\phi_{0} & =\left(\frac{2}{x}\right)^{\nu} \Gamma(1+\nu) \\
\phi_{m} & =\frac{(\nu+2 m)(\nu+m-1)}{m(\nu+2 m-2)} \phi_{m-1} .
\end{aligned}
$$


3. The Irregular Bessel Function. The irregular Bessel function $Y_{\nu}(x)$ is defined as

$$
Y_{\nu}(x)=\frac{J_{\nu}(x) \cos \nu \pi-J_{-\nu}(x)}{\sin \nu \pi} .
$$

Expansions for $J_{-\nu}(x)$ in terms of $J_{\nu+m}(x)$ are readily obtained [2], for example one may write:

$$
\begin{aligned}
J_{-\nu}(x)=\left(\frac{x}{2}\right)^{-2 \nu} \Gamma(1-2 \nu) \sum_{m=0}^{\infty} & \frac{(\nu+2 m)}{m !} \\
& \cdot \frac{\Gamma(\nu+m)}{\Gamma(1-m-2 \nu)} \frac{1}{\Gamma(1+m-\nu)} J_{\nu+2 m}(x) .
\end{aligned}
$$

Substitution of Eq. (14) into Eq. (13) yields the result that

$$
Y_{\nu}(x)=\sum_{m=0}^{\infty} \gamma_{m} J_{\nu+2 m}(x)
$$

where

$$
\begin{aligned}
& \gamma_{0}=\cot \nu \pi-\frac{1}{\pi}\left(\frac{2}{x}\right)^{2 \nu} \frac{\Gamma^{2}(1+\nu)}{\nu}, \\
& \gamma_{1}=\left(\frac{2}{\pi}\right)\left(\frac{2}{x}\right)^{2 \nu}\left(\frac{\nu+2}{1-\nu}\right) \Gamma^{2}(1+\nu), \\
& \gamma_{m}=-\frac{(\nu+2 m)(2 \nu+m-1)(\nu+m-1)}{m(m-\nu)(\nu+2 m-2)} \gamma_{m-1} .
\end{aligned}
$$

For small values of $|\nu|$ the coefficient $\gamma_{0}$ may be expanded as

$$
\begin{aligned}
\gamma_{0}=\frac{2}{\pi}\left[A-\nu\left(\frac{S_{2}}{2}\right.\right. & \left.+A^{2}+\frac{\pi^{2}}{6}\right)+\nu^{2}\left(\frac{S_{3}}{3}+A S_{2}+\frac{2 A^{3}}{3}\right) \\
& \left.-\nu^{3}\left(\frac{S_{4}}{4}+\frac{2 A S_{3}}{3}+\frac{S_{2}^{2}}{4}+A^{2} S_{2}+\frac{A^{4}}{3}+\frac{\pi^{4}}{90}\right)+\cdots\right],
\end{aligned}
$$

where

$$
A=0.5772156649 \cdots+\log \frac{x}{2}
$$

and

$$
\begin{aligned}
& S_{n}=\sum_{p=1}^{\infty} p^{-n}, \\
& S_{2}=\frac{\pi^{2}}{6}, \\
& S_{3}=1.202056903 \cdots, \\
& S_{4}=\frac{\pi^{4}}{90} .
\end{aligned}
$$


Thus, if we have obtained $J_{\nu+n}(x)$, we may use Eq. (15) to calculate $Y_{\nu}(x)$. The Wronskian relation,

$$
Y_{\nu}(x) J_{\nu+1}(x)-Y_{\nu+1}(x) J_{\nu}(x)=\frac{2}{\pi x},
$$

gives $Y_{\nu+1}(x)$. From $Y_{\nu}(x), Y_{\nu+1}(x)$ one may obtain the values of $Y_{\nu+n}(x), n>1$, by recurrence, Eq. (1).

If $x$ is close to a zero of $J_{\nu}(x)$, then Eq. (17) does not provide a suitable method for obtaining $Y_{v+1}(x)$. To avoid this difficulty one may obtain a series for $Y_{v+1}(x)$ which is analogous to the series for $Y_{\nu}(x)$, Eq. (15). This is readily accomplished by differentiating Eq. (15) and using the relation:

$$
Y_{\nu+1}(x)=\frac{\nu}{x} Y_{\nu}(x)-\frac{d Y_{\nu}}{d x}(x) \text {. }
$$

After some manipulation one may obtain the result

$$
Y_{\nu+1}=\sum_{m=0}^{\infty} \xi_{m} J_{\nu+m}
$$

where

$$
\begin{aligned}
\xi_{0} & =-\frac{1}{\pi}\left(\frac{2}{x}\right)^{1+2 \nu} \Gamma^{2}(1+\nu), \\
\xi_{1} & =\gamma_{0}-\frac{1}{2} \gamma_{1}, \\
\xi_{2 m} & =\frac{3 \nu}{x} \gamma_{m}, \quad m \geq 1, \\
\xi_{2 m+1} & =\frac{1}{2}\left(\gamma_{m}-\gamma_{m+1}\right), \quad m \geqq 1,
\end{aligned}
$$

and the $\gamma_{m}$ are as defined by Eq. (15).

4. Bessel Functions of Imaginary Argument. Analogous formulae are easily derived for the Bessel functions of imaginary argument $I_{\nu}(x)$ cind $K_{\nu}(x)$. The regular function $I_{\nu}(x)$ obeys the recurrence relation

$$
I_{\nu-1}(x)-I_{\nu+1}(x)=\frac{2 \nu}{x} I_{\nu}(x) .
$$

The irregular function $K_{\nu}(x)$ obeys the relation

$$
K_{\nu-1}(x)-K_{\nu+1}(x)=-\frac{2 \nu}{x} K_{\nu}(x) .
$$

It is convenient to define the function $\bar{K}_{\nu}(x)=(-1)^{\nu} K_{\nu}(x)$. Then $I_{\nu}(x)$ and $\bar{K}_{\nu}(x)$ both obey the same relation, viz:

$$
G_{\nu-1}(x)-G_{\nu+1}(x)=\frac{2 \nu}{x} G_{\nu}(x)
$$


One readily sees then that taking

$$
\begin{aligned}
& 0=G_{\nu+M+1}=\alpha I_{\nu+M+1}(x)+\beta \bar{K}_{\nu+M+1}(x) \\
& a=G_{\nu+M}=\alpha I_{\nu+M}(x)+\beta \bar{K}_{\nu+M}(x)
\end{aligned}
$$

will once again yield

$$
G_{\nu+n}(x) \cong \alpha I_{\nu+n}(x),
$$

to any desired accuracy for $M \gg N \geqq n$. The addition theorem analogous to Eq. (8) is

$$
2^{\nu} \sum_{m=0}^{\infty}(-1)^{m}(\nu+2 m) I_{\imath+2 m}(x) x^{-\nu} \Gamma(\nu+m) / m !=1 .
$$

In order to avoid the use of an alternating series, however, it proves useful to use a different addition theorem [2], viz:

$$
2\left(\frac{2}{x}\right)^{\nu} \frac{\Gamma(1+\nu)}{\Gamma(1+2 \nu)} \sum_{m=0}^{\infty} \frac{(\nu+m)}{m !} \Gamma(2 \nu+m)\left(e^{-x} I_{\nu+m}(x)\right)=1 .
$$

This leads to the following formula for $\alpha$ :

$$
\alpha \cong \sum_{m=0}^{L} \psi_{m} G_{\nu+m}(x),
$$

where

$$
\begin{aligned}
\psi_{0} & =e^{-x}\left(\frac{2}{x}\right)^{\nu} \Gamma(1+\nu) \\
\psi_{m} & =\frac{(\nu+m)(2 \nu+m-1)}{m(\nu+m-1)} \psi_{m-1} .
\end{aligned}
$$

The irregular function $K_{\nu}(x)$ may be treated completely analogously to $Y_{\nu}(x)$. The irregular function $K_{\nu}(x)$ is defined as

$$
K_{\nu}(x)=\frac{\pi}{2}\left[\frac{I_{-\nu}(x)-I_{\nu}(x)}{\sin \nu \pi}\right] .
$$

By means of the expansion

$$
\begin{aligned}
I_{-\nu}(x)=\left(\frac{x}{2}\right)^{-2 \nu} \Gamma(1-2 \nu) \sum_{m=0}^{\infty}(-1)^{m} \frac{(\nu+2 m)}{m !} & \frac{\Gamma(\nu+m)}{\Gamma(1-m-2 \nu)} \\
& \cdot \frac{1}{\Gamma(1+m-\nu)} I_{\nu+2 m}(x),
\end{aligned}
$$

one obtains the result that

$$
K_{\nu}(x)=\sum_{m=0}^{\infty} \delta_{m} I_{\nu+2 m}(x),
$$

where

$$
\delta_{0}=-\frac{1}{2 \nu}\left[\frac{\nu \pi}{\sin \nu \pi}-\left(\frac{2}{x}\right)^{2 \nu} \Gamma^{2}(1+\nu)\right]
$$




$$
\begin{aligned}
\delta_{1} & =\Gamma^{2}(1+\nu)\left(\frac{2}{x}\right)^{2 \nu} \frac{(\nu+2)}{(1-\nu)} \\
\delta_{m} & =\frac{(\nu+2 m)(2 \nu+m-1)(\nu+m-1)}{m(\nu+2 m-2)(m-\nu)} \delta_{m+1} .
\end{aligned}
$$

For small values of $|\nu|$ the coefficient $\delta_{0}$ may be expanded as

$$
\begin{aligned}
\delta_{0}=-\left[A-\nu\left(\frac{S_{2}}{2}\right.\right. & \left.+A^{2}-\frac{\pi^{2}}{12}\right)+\nu^{2}\left(\frac{S_{3}}{3}+A S_{2}+\frac{2 A^{3}}{3}\right) \\
& \left.-\nu^{3}\left(\frac{S_{4}}{4}+\frac{2 A S_{3}}{3}+\frac{S_{2}{ }^{2}}{4}+A^{2} S_{2}+\frac{A^{4}}{3}-\frac{7 \pi^{4}}{720}\right)+\cdots\right],
\end{aligned}
$$

where $A$ and $S_{n}$ are defined as in Eq. (16).

Thus, as before, if we have obtained $I_{\nu+n}(x)$ we may use Eq. (30) to calculate $K_{\nu}(x)$.

The Wronskian relation,

$$
K_{\nu}(x) I_{\nu+1}(x)+K_{\nu+1}(x) I_{\nu}(x)=\frac{1}{x},
$$

gives $K_{\nu+1}(x)$. From $K_{\nu}(x), K_{\nu+1}(x)$ one may obtain the values of $K_{\nu+n}(x)$ for $n>1$ by recurrence Eq. (21). Unlike $J_{\nu}, I_{\nu}(x)$ is not an oscillatory function and the Wronskian can, therefore, serve to give $K_{\nu+1}(x)$ without difficulty.

However, Eq. (30) does not yield high accuracy for $x \gg 1$, since for large $x$

$$
\delta_{0} I_{\nu} \sim-\sum_{m=1}^{\infty} \delta_{m} I_{\nu+2 m}
$$

This difficulty can, in practice, be overcome by the evaluation [3], [4] of the integral representation of $K_{\nu}(x)$

$$
K_{\nu}(x)=\int_{0}^{\infty} e^{-x \cosh y} \cosh \nu y d y
$$

5. Large Values of the Argument. If the values and derivatives of any one of the functions $J_{\nu}(x), Y_{\nu}(x), I_{\nu}(x), K_{\nu}(x)$ are readily obtained, then the above techniques are somewhat too cumbersome. In particular, if one has $Y_{\nu}(x)$, $Y_{\nu+1}(x)\left[K_{\nu}(x), K_{\nu+1}(x)\right]$ then one may obtain the values of $Y_{\nu+n}(x)\left[K_{\nu+n}(x)\right]$ by a straightforward recurrence. On the other hand, if one has $J_{\nu}(x)\left[I_{\nu}(x)\right]$, then one may follow the procedure of Eqs. (2-7) [Eqs. (23-24)]. However, now $\alpha$ is given simply by

$$
\alpha=F_{\nu}(x) / J_{\nu}(x)
$$

for $J_{\nu}(x)$, or

$$
\alpha=G_{\nu}(x) / I_{\nu}(x)
$$

for $I_{\nu}(x)$.

For $x \geqq 10$ the necessary values and derivatives of the functions $J_{\nu}(x), Y_{\nu}(x)$, $I_{\nu}(x), K_{\nu}(x)$ are easily obtained by the phase-amplitude method [5] for $0 \leqq \nu<1$. 
For very large values of the argument, this technique is more suitable for computations than the methods outlined in the previous sections.

A subroutine [6] for a high speed calculating machine, the IBM 704, has been written incorporating the methods described here for the calculation of Bessel functions.

New York University, New York City, New York and Los Alamos Scientific Laboratory, Los Alamos, New Mexico

Massachusetts Institute of Technology, Cambridge, Massachusetts and Los Alamos Scientific Laboratory, Los Alamos, New Mexico

1. British Association for the Advancement of Science, "Bessel functions, Part II," Mathematical Tables, Cambridge University Press, 1952. An application of a recurrence technique to the calculation of $I_{n}(x)$ is credited in the Introduction to J. C. P. Miller. An extension of this technique for use on high speed computers for the calculation of Bessel functions of integral and half integral order appeared in print after the completion of this manuscript. I. A. Stegun \& M. Abramowitz, "Generation of Bessel functions on high speed computers," MTAC, v. XI, 1957, p. 255.

2. G. N. Watson, A Treatise on the Theory of Bessel Functions, Second Edition, Cambridge University Press, 1948 , p. 139 and 369.

3. Y. L. LUKE, "Simple formulas for the evaluation of some higher transcendental functions," Journal of Math. and Phys., v. 34, 1956, p. 298-307.

4. H. E. Fetris, "Numerical calculation of certain definite integrals by Poisson's summation formula," MTAC, v. IX, 1955, p. 85-92.

5. M. Goldstein \& R. M. THALER, "Bessel functions for large arguments;" MTAC, v. XII, 1958, p. 18-26.

6. M. Goldstein \& M. Kresge, NU BES I, Share Distribution 469, Share Program Librarian, IBM, 590 Madison Avenue, New York 22, New York. 